\title{
The Center Conditions and Bifurcation of Limit Cycles at the Degenerate Singularity of a Three-Dimensional System
}

\author{
Shugang Song, ${ }^{1}$ Jingjing Feng, ${ }^{1}$ and Qinlong Wang ${ }^{2}$ \\ ${ }^{1}$ School of Information and Mathematics, Yangtze University, Jingzhou 434023, China \\ ${ }^{2}$ School of Science, Hezhou University, Hezhou 542899, China \\ Correspondence should be addressed to Qinlong Wang; wqinlong@163.com
}

Received 25 April 2014; Revised 23 June 2014; Accepted 26 June 2014; Published 9 July 2014

Academic Editor: Junjie Wei

Copyright (c) 2014 Shugang Song et al. This is an open access article distributed under the Creative Commons Attribution License, which permits unrestricted use, distribution, and reproduction in any medium, provided the original work is properly cited.

\begin{abstract}
We investigate multiple limit cycles bifurcation and center-focus problem of the degenerate equilibrium for a three-dimensional system. By applying the method of symbolic computation, we obtain the first four quasi-Lyapunov constants. It is proved that the system can generate 3 small limit cycles from nilpotent critical point on center manifold. Furthermore, the center conditions are found and as weak foci the highest order is proved to be the fourth; thus we obtain at most 3 small limit cycles from the origin via local bifurcation. To our knowledge, it is the first example of multiple limit cycles bifurcating from a nilpotent singularity for the flow of a high-dimensional system restricted to the center manifold.
\end{abstract}

\section{Introduction}

About dynamical behavior of the trajectories of threedimensional system, bifurcation of limit cycles is one of major concerns; particularly, for Hopf bifurcation of a nondegenerate equilibrium with a pair of pure imaginary roots and a negative one, many investigations have been carried out in the past decades, for example, [1-4] for the three-dimensional chaotic systems, [5-8] for the three-dimensional Lotka-Volterra systems, and [9] for general three-dimensional systems.

However, up till now, study on bifurcation of limit cycles from the degenerate singularity for high-dimensional nonlinear dynamical systems is hardly seen in published references. In this paper, we investigate the following threedimensional systems:

$$
\begin{gathered}
\frac{\mathrm{d} x}{\mathrm{~d} t}=y+\mu x^{2}+\sum_{k+2 j+2 l=3}^{\infty} A_{k j l} x^{k} y^{j} u^{l}=X(x, y, u), \\
\frac{\mathrm{d} y}{\mathrm{~d} t}=-2 x^{3}+2 \mu x y+\sum_{k+2 j+2 l=4}^{\infty} B_{k j l} x^{k} y^{j} u^{l}=Y(x, y, u), \\
\frac{\mathrm{d} u}{\mathrm{~d} t}=-d u+\sum_{k+j+l=2}^{\infty} d_{k j l} x^{k} y^{j} u^{l}=U(x, y, u),
\end{gathered}
$$

where $x, y, u, t, d, A_{k j l}, B_{k j l}, d_{k j l} \in \mathbb{R}(d>0, k, j, l \in \mathbb{N})$. Obviously system (1) has the Jacobian matrix at the origin as follows:

$$
A=\left(\begin{array}{cc}
A_{1} & 0 \\
0 & -d
\end{array}\right), \quad \text { where } A_{1}=\left(\begin{array}{ll}
0 & 1 \\
0 & 0
\end{array}\right) .
$$

One notices that the origin has two zero eigenvalues and one negative eigenvalue and the block matrix $A_{1}$ is nilpotent. From the center manifold theorem, for the system (1), there exists the center manifold: $u=u(x, y)$ with $u(0,0)=0$, $\mathrm{D} u(0,0)=0$, and more the flow on the center manifold is governed by $a / a$

$$
\left(\begin{array}{c}
\dot{x} \\
\dot{y}
\end{array}\right)=A_{1}\left(\begin{array}{l}
x \\
y
\end{array}\right)+\left(\begin{array}{c}
X(x, y, u(x, y))-y \\
Y(x, y, u(x, y))
\end{array}\right),
$$

namely,

$$
\begin{gathered}
\frac{\mathrm{d} x}{\mathrm{~d} t}=y+\mu x^{2}+\sum_{k+2 j=3}^{\infty} a_{k j} x^{k} y^{j}=\widetilde{X}(x, y), \\
\frac{\mathrm{d} y}{\mathrm{~d} t}=-2 x^{3}+2 \mu x y+\sum_{k+2 j=4}^{\infty} b_{k j} x^{k} y^{j}=\widetilde{Y}(x, y),
\end{gathered}
$$


where $x, y, t, a_{k j}, b_{k j} \in \mathbb{R}(k, j \in \mathbb{N})$. It is usually called the reduced system and system (1) is topological equivalent to system (4) in the vicinity of the origin [10]. Thus by investigating system (4), we can solve Hopf bifurcation of the origin on center manifold of the three-dimensional system (1). At the same time, we also discuss the centerfocus problem for the flow restricted to the center manifold, which closely relates to the maximum number of limit cycles bifurcating from the origin.

As far as investigation about limit cycles bifurcation of a nilpotent critical point in planar system is concerned, it is similar to nondegenerate case that detecting nilpotent center and calculating the focal value are needed [11]. There exist some available and classical ways, for instance, Poincare return map [12], Lyapunov function [13], and the normal form theory [14]. At the same time, some good results on the cyclicity were obtained $[11,12,15,16]$. Recently in $[17$, $18]$, the authors gave an inverse integral factor method of calculating the quasi-Lyapunov constants of the three-order nilpotent critical point; it is convenient to compute the higher order focal values and solve the center-focus problem of the equilibrium. Here we extend this method's application to the three-dimensional system (1) and consider its specific example as follows:

$$
\begin{gathered}
\frac{\mathrm{d} x}{\mathrm{~d} t}=y+a_{0} u x-2 x y=X, \\
\frac{\mathrm{d} y}{\mathrm{~d} t}=y^{2}-2 x^{3}+b_{0} u y=Y, \\
\frac{\mathrm{d} u}{\mathrm{~d} t}=-u+d_{1} x y=U,
\end{gathered}
$$

where $a_{0}, b_{0}, d_{1} \in \mathbb{R}$.

The rest of this paper is organized as follows. In Section 2, the corresponding quasi-Lyapunov constants are computed and the center conditions on the center manifold are determined. In Section 3, the multiple local bifurcations at the origin for system (5) are investigated, three limit cycles from the origin are obtained, and it is proved at most three small limit cycles from the origin via local bifurcation. In this work, the system and problem are all considered for the first time.

\section{Quasi-Lyapunov Constants and Center Conditions}

In this part, we firstly investigate the singular point quantities of the origin. For the center manifold of system (5), one can determine the formal expression $u=\widetilde{u}(x, y)$ and obtain the same form as system (4):

$$
\begin{aligned}
& \frac{\mathrm{d} x}{\mathrm{~d} t}=y+a_{0} \tilde{u} x-2 x y=\widetilde{X}, \\
& \frac{\mathrm{d} y}{\mathrm{~d} t}=y^{2}-2 x^{3}+b_{0} \tilde{u} y=\widetilde{Y} .
\end{aligned}
$$

Furthermore, we give the definition of quasi-Lyapunov constants for system (6) and the way of computing them; more details can be found in [17-19].
Lemma 1 (Theorems 8.7.1 and 8.7.2 in [17]). For system (6), any positive integer $s$ and a given number sequence $\left\{c_{0 \beta}\right\}, \beta \geqslant$ 3 , we can derive successively and uniquely the terms of the following formal series with the coefficients $c_{\alpha \beta}$ satisfying $\alpha \neq 0$,

$$
M(x, y)=y^{2}+\sum_{\alpha+\beta=3}^{\infty} c_{\alpha \beta} x^{\alpha} y^{\beta}
$$

such that

$$
\left(\frac{\partial X}{\partial x}+\frac{\partial Y}{\partial y}\right) M-(s+1)\left(\frac{\partial M}{\partial x} X+\frac{\partial M}{\partial y} Y\right)=\sum_{m=6}^{\infty} \omega_{m} x^{m} .
$$

And, if $\alpha \neq 0, c_{\alpha \beta}$ is determined by the following recursive formula:

$$
\begin{aligned}
c_{\alpha \beta}=\frac{1}{(s+1) \alpha} \sum_{k+j=2}^{\alpha+\beta-1}\{[ & k-(s+1)(\alpha-k)] a_{k j} c_{\alpha-k, \beta-j+1} \\
+ & {[j-(s+1)(\beta-j+2)] } \\
& \left.\times b_{k j} c_{\alpha-k-1, \beta-j+2}\right\}
\end{aligned}
$$

and, for any positive integer $m \geqslant 6, \omega_{m}$ is determined by the following recursive formula:

$$
\begin{array}{r}
\omega_{m}=\sum_{k+j=2}^{m-1}\left[(k-(s+1)(\alpha-k+1)) a_{k j} c_{m-k+1,-j}\right. \\
\left.+(j-(s+1)(1-j)) b_{k j} c_{m-k, 1-j}\right]
\end{array}
$$

and, when $\alpha<0$ or $\beta<0$, we have let $c_{\alpha \beta}=0$.

Particularly, by choosing appropriate $s$ and number sequence $\left\{c_{0 \beta}\right\}(\beta \geqslant 3)$, we can make $\omega_{2 i+1}(s)=0$ and then let

$$
\omega_{2 i+4}(s)=(2 i-4 s-1) \lambda_{i} .
$$

Definition 2. The $\lambda_{i}$ in (11) is called the $i$ th quasi-Lyapunov constant of the origin of system (6), $i=1,2, \ldots$, and more if all the quasi-Lyapunov constants vanish, that is, $\lambda_{m}=0$, $m=1,2, \ldots$, then the origin of system (5) is a center on the local center manifold at the origin.

Lemma 3. For system (6), the $m$ th focal value $v_{2 m}(-2 \pi)$ at the origin of system (6) is algebraic equivalent to $\sigma_{m} \lambda_{m}$; that is, for any positive integer $m=2,3, \ldots$, if $v_{2}=v_{4}=\cdots=v_{2 m-2}=0$ and $\lambda_{1}=\lambda_{2}=\cdots=\lambda_{m-1}=0$ hold, then $v_{2 m}=\sigma_{m} \lambda_{m}$, where $\sigma_{m}$ is a defined constant (which is given in $[17,18]$ ).

Remark 4. According to Lemma 1, one sees that each $\omega_{m}$ in (10) is related to only the coefficients of the first $m-1$ degree terms of system (6). Here we determine the above $\tilde{u}(x, y)$ only up to a tenth degree polynomial with respect to $x, y$ as follows:

$$
\begin{gathered}
\tilde{u}(x, y)=d_{1} y(x-y)+d_{1} y^{2}(x+y) \\
+u_{4}+u_{5}+\cdots+u_{10},
\end{gathered}
$$


where $u_{i}, i=4, \ldots, 10$ is a homogeneous polynomial in $x, y$ of degree $i$, respectively, (which can be seen in the Appendix); thus $\widetilde{X}$ and $\widetilde{Y}$ in system (6) are two polynomials with degree 11. And more all $a_{k j}, b_{k j}$ of $c_{\alpha \beta}$ in (9) are given definitely by the coefficients $a_{0}, b_{0}, d_{1}$ (the specific $c_{\alpha \beta}$ is available in Email address of the corresponding author); the $\omega_{m}$ in (10) is given by the following specific form:

$$
\begin{aligned}
\omega_{m}= & -32 d_{1}\left(686-94 a_{0} d_{1}-31 b_{0} d_{1}\right) \\
& \times\left(21 a_{0}+b_{0}-a_{0} m+10 a_{0} s-a_{0} m s\right) \\
& \times c[m-10,0] \\
& -2208 d_{1}\left(19 a_{0}+b_{0}-a_{0} m+9 a_{0} s-a_{0} m s\right) \\
& \times c[m-9,0] \\
& +4 d_{1}\left(144-11 a_{0} d_{1}-3 b_{0} d_{1}\right) \\
& \times\left(17 a_{0}+b_{0}-a_{0} m+8 a_{0} s-a_{0} m s\right) \\
& \times c[m-8,0] \\
& +40 d_{1}\left(15 a_{0}+b_{0}-a_{0} m+7 a_{0} s-a_{0} m s\right) \\
& \times c[m-7,0] \\
& -24 d_{1}\left(13 a_{0}+b_{0}-a_{0} m+6 a_{0} s-a_{0} m s\right) \\
& \times c[m-6,0] \\
& +2 d_{1}\left(9 a_{0}+b_{0}-a_{0} m+4 a_{0} s-a_{0} m s\right) \\
& \times c[m-4,0]+2(1+s) c[m-3,0] .
\end{aligned}
$$

Applying the powerful symbolic computation function of Mathematica system and the recursive formulas in Lemma 1, and from Remark 4, we obtain the first 7 quantities as follows:

$$
\begin{aligned}
& \omega_{6}=0 \\
& \omega_{7}=3(1+s) c_{03}, \\
& \omega_{8}=\frac{4}{5} d_{1}\left(2 a_{0}-b_{0}\right)(3-4 s), \\
& \omega_{9}=\frac{2}{3} a_{0} d_{1}(1-s), \\
& \omega_{10}=\frac{2}{7} a_{0} d_{1}\left(1-6 a_{0} d_{1}\right), \\
& \omega_{11}=\frac{1}{112}\left(73 a_{0} d_{1}-704 a_{0}^{2} d_{1}^{2}+840 c_{05}\right), \\
& \omega_{12}=-\frac{397}{105} a_{0} d_{1} .
\end{aligned}
$$

In the above expression of each $\omega_{k}, k=7, \ldots, 12$, we have already let $\omega_{6}=\cdots=\omega_{k-1}=0$.

Particularly, in order to make $\omega_{2 i+1}=0, i=1, \ldots, 5$, we let $s=1$ and choose

$$
c_{03}=0, \quad c_{05}=\frac{1}{840}\left(704 a_{0}^{2} d_{1}^{2}-73 a_{0} d_{1}\right) .
$$

Thus from the expression (11) in Lemma 1, we have the following.

Theorem 5. For the flow on center manifold of system (5), we get the first 4 quasi-Lyapunov constants of the origin as follows:

$$
\begin{aligned}
& \lambda_{1}=0, \\
& \lambda_{2}=\frac{4}{5} d_{1}\left(2 a_{0}-b_{0}\right), \\
& \lambda_{3}=\frac{2}{7} a_{0} d_{1}\left(1-6 a_{0} d_{1}\right), \\
& \lambda_{4}=-\frac{397}{315} a_{0} d_{1} .
\end{aligned}
$$

Theorem 6. For system (5), the origin is a center on the local center manifold if and only if the following condition is satisfied:

$$
d_{1}=0 \quad \text { or } \quad a_{0}=b_{0}=0 .
$$

Proof. The proof of the necessity is easy; then we omit it. Now we prove the sufficient condition; this technique derives from the method of Darboux (also see [20-23]). Obviously, if $d_{1}=0$ in the conditions (17) holds, then system (5) has the corresponding form as follows:

$$
\begin{gathered}
\frac{\mathrm{d} x}{\mathrm{~d} t}=y+a_{0} u x-2 x y, \\
\frac{\mathrm{d} y}{\mathrm{~d} t}=y^{2}-2 x^{3}+b_{0} u y, \quad \frac{\mathrm{d} u}{\mathrm{~d} t}=-u .
\end{gathered}
$$

And more we can figure out easily one algebraic invariant surface for system (18): $F(x, y, u)=u$; in fact, there exists a polynomial $K(x, y, u)=-1$, the cofactor of $F(x, y, u)$, such that $\mathrm{d} F /\left.\mathrm{d} t\right|_{(17)}=K F$. One can observe that $F(x, y, u)=$ 0 is tangent to the center eigenspace, the $(x, y)$-plane, at the origin. Thus it forms a local center manifold in a neighborhood of the origin. From $F(x, y, u)=u=0$, we substitute it into the first and second equations of the system defined by system (18) and we have the differential equations

$$
\frac{\mathrm{d} x}{\mathrm{~d} t}=y-2 x y, \quad \frac{\mathrm{d} y}{\mathrm{~d} t}=y^{2}-2 x^{3}
$$

which is a Hamiltonian system with Hamiltonian function: $H(x, y)=x^{4}-2 x y^{2}+y^{2}$. Therefore the origin is a center for systems (18) or (19) as shown in Figure 1(a).

And if $a_{0}=b_{0}=0$ in the conditions (17) holds, then system (5) has the corresponding form as follows:

$$
\frac{\mathrm{d} x}{\mathrm{~d} t}=y-2 x y, \quad \frac{\mathrm{d} y}{\mathrm{~d} t}=y^{2}-2 x^{3}, \quad \frac{\mathrm{d} u}{\mathrm{~d} t}=-u+d_{1} x y .
$$

Clearly, the above $H=x^{4}-2 x y^{2}+y^{2}$ is also a first integral of system (20); thus the origin is a center for the flow of system (5) restricted to a center manifold.

According to Lemma 3 and Theorem 6, then we have the following. 


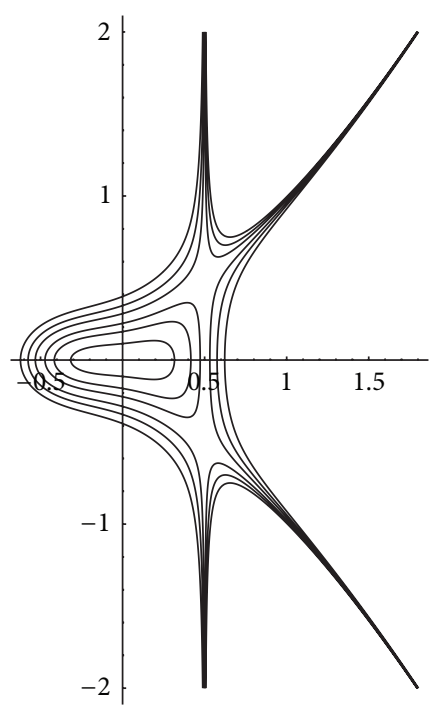

(a)

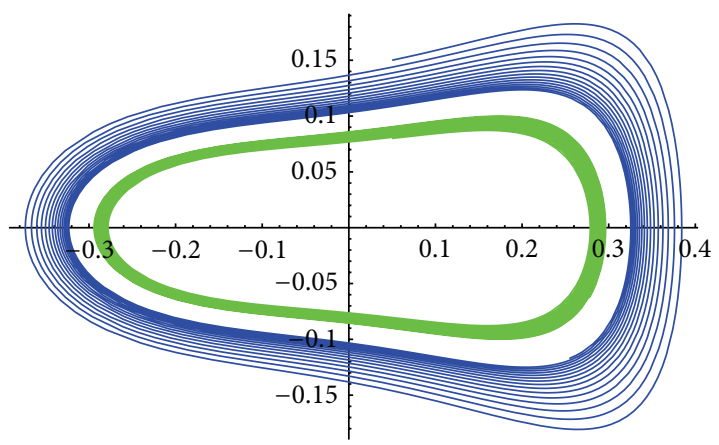

(b)

Figure 1: (a) The phase portrait of system (19) on the $(x, y)$-plane which shows that it is integrable at the origin; (b) the stable limit cycle bifurcated from the origin of system (24) with $\delta=0.001, d_{1}=1, a_{0}=1 / 6, b_{0}=1 / 3+0.001$.

Theorem 7. For the origin of system (5) as a weak focus on center manifold, the highest is the fourth order and its first 4 focal values are as follows:

$$
\begin{array}{ll}
v_{2}(-2 \pi)=\sigma_{1} \lambda_{1}, & v_{4}(-2 \pi)=\sigma_{2} \lambda_{2}, \\
v_{6}(-2 \pi)=\sigma_{3} \lambda_{3}, & v_{8}(-2 \pi)=\sigma_{4} \lambda_{4},
\end{array}
$$

where, for the expression of $v_{4}$, we have let $v_{2}=0$, and $v_{2}=$ $v_{4}=0$ for $v_{6}, v_{2}=v_{4}=v_{6}=0$ for $v_{8}$.

\section{Multiple Limit Cycle Bifurcation of the System}

In this section, we apply the focal values obtained in last section to discuss multiple bifurcation of the equilibrium and demonstrate there exist 3 and at most 3 limit cycles.

From Theorems 5 and 7, we have

Theorem 8. For the flow on center manifold of (5), the origin is a 4th-order fine focus; that is, $\lambda_{1}=\lambda_{2}=\lambda_{3}=0, \lambda_{4} \neq 0$ if and only if

$$
\begin{gathered}
b_{0}=2 a_{0}, \quad 6 a_{0} d_{1}=1 \\
\text { or } \quad b_{0}=\frac{1}{3 d_{1}}, \quad a_{0}=\frac{1}{6 d_{1}} \\
\left(d_{1} \neq 0\right) .
\end{gathered}
$$

Proof. Firstly, we prove the necessity, from the expressions (16) in Theorem 5; we let $\lambda_{1}=\lambda_{2}=\lambda_{3}=0$ and consider $\lambda_{4} \neq 0$ and then only $b_{0}=2 a_{0}$ and $6 a_{0} d_{1}=1$ are obtained at the same time. Then for the proof of the sufficiency, by substituting the above conditions into $\lambda_{i}, i=1,2,3,4$, one can get the conclusion easily. Furthermore we can transform the above conditions into the following form: $b_{0}=1 / 3 d_{1}$, $a_{0}=\left(1 / 6 d_{1}\right)\left(d_{1} \neq 0\right)$. Thus the proof is completed.

Remark 9. For the coefficients of system (5) or (6), when $d_{1} \neq 0$, there always exists a group of critical values: $a_{0}=$ $1 / 6 d_{1}, b_{0}=1 / 3 d_{1}$ such that the conditions (22) hold.

From Theorem 5, one calculates easily the Jacobian determinant with respect to the functions $\lambda_{2}, \lambda_{3}$ and variables $a_{0}$, $b_{0}$,

$$
J=\frac{\partial\left(\lambda_{2}, \lambda_{3}\right)}{\partial\left(a_{0} b_{0}\right)}=\frac{8}{35} d_{1}^{2}\left(1-12 a_{0} d_{1}\right) .
$$

Considering the conditions (22) and when $d_{1} \neq 0$ holds, we obtain $J=-(8 / 35) d_{1}^{2} \neq 0$. At the same time, one can get that $\lambda_{4}=-(397 / 1890) \neq 0$ holds.

Thus we take some appropriate perturbations for the coefficients of system (6) and get the perturbed system with the following form:

$$
\begin{gathered}
\frac{\mathrm{d} x}{\mathrm{~d} t}=\delta x+y+a_{0} \tilde{u} x-2 x y, \\
\frac{\mathrm{d} y}{\mathrm{~d} t}=2 \delta y+y^{2}-2 x^{3}+b_{0} \tilde{u} y,
\end{gathered}
$$

where $0<\delta \ll 1$. According to Theorem 2.2 in [18], system (6) has 3 limit cycles in the neighborhood of the origin; that is, system (5) has 3 limit cycles on the center manifold necessarily. One can refer to $[9,17,18]$ for more details about constructing of limit cycles. And using numerical method, we get an example of one stable limit cycle for system (24) as shown in Figure 1(b).

Therefore, from the above discussion and Theorem 7, we have the following. 
Theorem 10. For system (5), 3 and at most 3 small amplitude limit cycles can be bifurcated from the origin on the center manifold.

\section{Conclusions}

In summary, based on precise symbolic computation, we have investigated deeply multiple limit cycles bifurcation in the vicinity of the degenerate equilibrium for a threedimensional system. Firstly by computing its quasi-Lyapunov constants, we solved its center-focus problem on center manifold; thus the center conditions are found and as weak foci the highest order is proved to be the fourth. Then we obtained that the system can generate 3 small limit cycles from nilpotent critical point and at most 3 small limit cycles from the origin via local bifurcation. For the flow of a highdimensional system restricted to the center manifold, only one interesting example of multiple limit cycles bifurcating from its nilpotent singularity was given here, but we believe that more outcomes on this problem will be shown in the near future.

\section{Appendix}

Consider

$$
\begin{aligned}
u_{4}=-d_{1}( & -2 x^{4}+12 x^{3} y-36 x^{2} y^{2}+a_{0} d_{1} x^{2} y^{2} \\
& +b_{0} d_{1} x^{2} y^{2}+72 x y^{3}-3 a_{0} d_{1} x y^{3} \\
& \left.-5 b_{0} d_{1} x y^{3}-69 y^{4}+3 a_{0} d_{1} y^{4}+7 b_{0} d_{1} y^{4}\right), \\
u_{5}=d_{1} y( & 20 x^{4}-134 x^{3} y+474 x^{2} y^{2}-4 a_{0} d_{1} x^{2} y^{2} \\
& -5 b_{0} d_{1} x^{2} y^{2}-876 x y^{3}+5 a_{0} d_{1} x y^{3} \\
& \left.+5 b_{0} d_{1} x y^{3}+600 y^{4}+7 a_{0} d_{1} y^{4}+28 b_{0} d_{1} y^{4}\right), \\
u_{6}=-d_{1}( & 24 x^{6}-288 x^{5} y+14 a_{0} d_{1} x^{5} y+6 b_{0} d_{1} x^{5} y \\
& +1732 x^{4} y^{2}-144 a_{0} d_{1} x^{4} y^{2}-88 b_{0} d_{1} x^{4} y^{2} \\
& -6944 x^{3} y^{3}+744 a_{0} d_{1} x^{3} y^{3}+552 b_{0} d_{1} x^{3} y^{3} \\
& -3 a_{0}^{2} d_{1}^{2} x^{3} y^{3}-6 a_{0} b_{0} d_{1}^{2} x^{3} y^{3}-3 b_{0}^{2} d_{1}^{2} x^{3} y^{3} \\
& +20358 x^{2} y^{4}-2443 a_{0} d_{1} x^{2} y^{4}-2081 b_{0} d_{1} x^{2} y^{4} \\
& +17 a_{0}^{2} d_{1}^{2} x^{2} y^{4}+46 a_{0} b_{0} d_{1}^{2} x^{2} y^{4}+33 b_{0}^{2} d_{1}^{2} x^{2} y^{4} \\
& -42468 x y^{5}+5038 a_{0} d_{1} x y^{5}+4882 b_{0} d_{1} x y^{5} \\
& -40 a_{0}^{2} d_{1}^{2} x y^{5}-134 a_{0} b_{0} d_{1}^{2} x y^{5}-126 b_{0}^{2} d_{1}^{2} x y^{5} \\
& +45468 y^{6}-5003 a_{0} d_{1} y^{6}-5153 b_{0} d_{1} y^{6} \\
& \left.+40 a_{0}^{2} d_{1}^{2} y^{6}+152 a_{0} b_{0} d_{1}^{2} y^{6}+168 b_{0}^{2} d_{1}^{2} y^{6}\right),
\end{aligned}
$$

$$
\begin{aligned}
& u_{7}=d_{1}\left(40 x^{7}-1104 x^{6} y+12060 x^{5} y^{2}\right. \\
& -260 a_{0} d_{1} x^{5} y^{2}-128 b_{0} d_{1} x^{5} y^{2}-77700 x^{4} y^{3} \\
& +2860 a_{0} d_{1} x^{4} y^{3}+1700 b_{0} d_{1} x^{4} y^{3}+337632 x^{3} y^{4} \\
& -15498 a_{0} d_{1} x^{3} y^{4}-10704 b_{0} d_{1} x^{3} y^{4}+24 a_{0}^{2} d_{1}^{2} x^{3} y^{4} \\
& +56 a_{0} b_{0} d_{1}^{2} x^{3} y^{4}+33 b_{0}^{2} d_{1}^{2} x^{3} y^{4}-1012896 x^{2} y^{5} \\
& +49266 a_{0} d_{1} x^{2} y^{5}+39042 b_{0} d_{1} x^{2} y^{5}-94 a_{0}^{2} d_{1}^{2} x^{2} y^{5} \\
& -251 a_{0} b_{0} d_{1}^{2} x^{2} y^{5}-169 b_{0}^{2} d_{1}^{2} x^{2} y^{5}+1898388 x y^{6} \\
& -84891 a_{0} d_{1} x y^{6}-72276 b_{0} d_{1} x y^{6}+66 a_{0}^{2} d_{1}^{2} x y^{6} \\
& +67 a_{0} b_{0} d_{1}^{2} x y^{6}-166 b_{0}^{2} d_{1}^{2} x y^{6}-1625580 y^{7} \\
& +54873 a_{0} d_{1} y^{7}+45075 b_{0} d_{1} y^{7}+174 a_{0}^{2} d_{1}^{2} y^{7} \\
& \left.+915 a_{0} b_{0} d_{1}^{2} y^{7}+1419 b_{0}^{2} d_{1}^{2} y^{7}\right), \\
& u_{8}=-d_{1}\left(-576 x^{8}+44 a_{0} d_{1} x^{8}+12 b_{0} d_{1} x^{8}\right. \\
& +10976 x^{7} y-1264 a_{0} d_{1} x^{7} y-496 b_{0} d_{1} x^{7} y \\
& -106352 x^{6} y^{2}+16048 a_{0} d_{1} x^{6} y^{2} \\
& +7696 b_{0} d_{1} x^{6} y^{2} \\
& -114 a_{0}^{2} d_{1}^{2} x^{6} y^{2}-116 a_{0} b_{0} d_{1}^{2} x^{6} y^{2} \\
& -34 b_{0}^{2} d_{1}^{2} x^{6} y^{2}+704496 x^{5} y^{3} \\
& -126632 a_{0} d_{1} x^{5} y^{3}-69528 b_{0} d_{1} x^{5} y^{3} \\
& +1700 a_{0}^{2} d_{1}^{2} x^{5} y^{3}+2172 a_{0} b_{0} d_{1}^{2} x^{5} y^{3} \\
& +816 b_{0}^{2} d_{1}^{2} x^{5} y^{3}-3558660 x^{4} y^{4} \\
& +710088 a_{0} d_{1} x^{4} y^{4}+428808 b_{0} d_{1} x^{4} y^{4} \\
& -12764 a_{0}^{2} d_{1}^{2} x^{4} y^{4}-19064 a_{0} b_{0} d_{1}^{2} x^{4} y^{4} \\
& -8340 b_{0}^{2} d_{1}^{2} x^{4} y^{4}+14 a_{0}^{3} d_{1}^{3} x^{4} y^{4} \\
& +42 a_{0}^{2} b_{0} d_{1}^{3} x^{4} y^{4}+42 a_{0} b_{0}^{2} d_{1}^{3} x^{4} y^{4} \\
& +14 b_{0}^{3} d_{1}^{3} x^{4} y^{4}+14104992 x^{3} y^{5} \\
& -2960538 a_{0} d_{1} x^{3} y^{5}-1907570 b_{0} d_{1} x^{3} y^{5} \\
& +61685 a_{0}^{2} d_{1}^{2} x^{3} y^{5}+103855 a_{0} b_{0} d_{1}^{2} x^{3} y^{5} \\
& +50130 b_{0}^{2} d_{1}^{2} x^{3} y^{5}-125 a_{0}^{3} d_{1}^{3} x^{3} y^{5} \\
& -463 a_{0}^{2} b_{0} d_{1}^{3} x^{3} y^{5}-591 a_{0} b_{0}^{2} d_{1}^{3} x^{3} y^{5} \\
& -261 b_{0}^{3} d_{1}^{3} x^{3} y^{5}-43327872 x^{2} y^{6} \\
& +9065880 a_{0} d_{1} x^{2} y^{6}+6166752 b_{0} d_{1} x^{2} y^{6}
\end{aligned}
$$




$$
\begin{aligned}
& -200895 a_{0}^{2} d_{1}^{2} x^{2} y^{6}-374342 a_{0} b_{0} d_{1}^{2} x^{2} y^{6} \\
& -196423 b_{0}^{2} d_{1}^{2} x^{2} y^{6}+504 a_{0}^{3} d_{1}^{3} x^{2} y^{6} \\
& +2186 a_{0}^{2} b_{0} d_{1}^{3} x^{2} y^{6}+3360 a_{0} b_{0}^{2} d_{1}^{3} x^{2} y^{6} \\
& +1854 b_{0}^{3} d_{1}^{3} x^{2} y^{6}+94249296 x y^{7} \\
& -18564504 a_{0} d_{1} x y^{7}-13274868 b_{0} d_{1} x y^{7} \\
& +412530 a_{0}^{2} d_{1}^{2} x y^{7}+833201 a_{0} b_{0} d_{1}^{2} x y^{7} \\
& +468601 b_{0}^{2} d_{1}^{2} x y^{7}-1097 a_{0}^{3} d_{1}^{3} x y^{7} \\
& -5317 a_{0}^{2} b_{0} d_{1}^{3} x y^{7}-9303 a_{0} b_{0}^{2} d_{1}^{3} x y^{7} \\
& -6011 b_{0}^{3} d_{1}^{3} x y^{7}-105628356 y^{8} \\
& +18948615 a_{0} d_{1} y^{8}+13977981 b_{0} d_{1} y^{8} \\
& -411312 a_{0}^{2} d_{1}^{2} y^{8}-868420 a_{0} b_{0} d_{1}^{2} y^{8} \\
& -503532 b_{0}^{2} d_{1}^{2} y^{8}+1097 a_{0}^{3} d_{1}^{3} y^{8} \\
& +5673 a_{0}^{2} b_{0} d_{1}^{3} y^{8}+10687 a_{0} b_{0}^{2} d_{1}^{3} y^{8} \\
& \left.+7551 b_{0}^{3} d_{1}^{3} y^{8}\right) \\
& u_{9}=d_{1}\left(-2208 x^{9}+77328 x^{8} y-2384 a_{0} d_{1} x^{8} y\right. \\
& -784 b_{0} d_{1} x^{8} y-1227512 x^{7} y^{2} \\
& +64396 a_{0} d_{1} x^{7} y^{2}+26272 b_{0} d_{1} x^{7} y^{2} \\
& +12357160 x^{6} y^{3}-835460 a_{0} d_{1} x^{6} y^{3} \\
& -396608 b_{0} d_{1} x^{6} y^{3}+3144 a_{0}^{2} d_{1}^{2} x^{6} y^{3} \\
& +3498 a_{0} b_{0} d_{1}^{2} x^{6} y^{3}+1096 b_{0}^{2} d_{1}^{2} x^{6} y^{3} \\
& -89203392 x^{5} y^{4}+6916384 a_{0} d_{1} x^{5} y^{4} \\
& +3676396 b_{0} d_{1} x^{5} y^{4}-49214 a_{0}^{2} d_{1}^{2} x^{5} y^{4} \\
& -63780 a_{0} b_{0} d_{1}^{2} x^{5} y^{4}-22834 b_{0}^{2} d_{1}^{2} x^{5} y^{4} \\
& +483032256 x^{4} y^{5}-40282356 a_{0} d_{1} x^{4} y^{5} \\
& -23266264 b_{0} d_{1} x^{4} y^{5}+378254 a_{0}^{2} d_{1}^{2} x^{4} y^{5} \\
& +553724 a_{0} b_{0} d_{1}^{2} x^{4} y^{5}+217218 b_{0}^{2} d_{1}^{2} x^{4} y^{5} \\
& -180 a_{0}^{3} d_{1}^{3} x^{4} y^{5}-607 a_{0}^{2} b_{0} d_{1}^{3} x^{4} y^{5} \\
& -687 a_{0} b_{0}^{2} d_{1}^{3} x^{4} y^{5}-261 b_{0}^{3} d_{1}^{3} x^{4} y^{5} \\
& -1968992136 x^{3} y^{6}+169196340 a_{0} d_{1} x^{3} y^{6} \\
& +104279988 b_{0} d_{1} x^{3} y^{6}-1780906 a_{0}^{2} d_{1}^{2} x^{3} y^{6} \\
& -2890212 a_{0} b_{0} d_{1}^{2} x^{3} y^{6}-1233114 b_{0}^{2} d_{1}^{2} x^{3} y^{6}
\end{aligned}
$$

$$
\begin{aligned}
& +1292 a_{0}^{3} d_{1}^{3} x^{3} y^{6}+4961 a_{0}^{2} b_{0} d_{1}^{3} x^{3} y^{6} \\
& +6477 a_{0} b_{0}^{2} d_{1}^{3} x^{3} y^{6}+2877 b_{0}^{3} d_{1}^{3} x^{3} y^{6} \\
& +5820320664 x^{2} y^{7}-495558804 a_{0} d_{1} x^{2} y^{7} \\
& -321861864 b_{0} d_{1} x^{2} y^{7}+5224500 a_{0}^{2} d_{1}^{2} x^{2} y^{7} \\
& +9158682 a_{0} b_{0} d_{1}^{2} x^{2} y^{7}+4161294 b_{0}^{2} d_{1}^{2} x^{2} y^{7} \\
& -3274 a_{0}^{3} d_{1}^{3} x^{2} y^{7}-12840 a_{0}^{2} b_{0} d_{1}^{3} x^{2} y^{7} \\
& -16145 a_{0} b_{0}^{2} d_{1}^{3} x^{2} y^{7}-5819 b_{0}^{3} d_{1}^{3} x^{2} y^{7} \\
& -11169394848 x y^{8}+901925700 a_{0} d_{1} x y^{8} \\
& +606394284 b_{0} d_{1} x y^{8}-8530348 a_{0}^{2} d_{1}^{2} x y^{8} \\
& -15428426 a_{0} b_{0} d_{1}^{2} x y^{8}-6980247 b_{0}^{2} d_{1}^{2} x y^{8} \\
& +949 a_{0}^{3} d_{1}^{3} x y^{8}-2716 a_{0}^{2} b_{0} d_{1}^{3} x y^{8} \\
& -23065 a_{0} b_{0}^{2} d_{1}^{3} x y^{8}-31601 b_{0}^{3} d_{1}^{3} x y^{8} \\
& +10324368000 y^{9}-750336780 a_{0} d_{1} y^{9} \\
& -509164044 b_{0} d_{1} y^{9}+5239852 a_{0}^{2} d_{1}^{2} y^{9} \\
& +8941749 a_{0} b_{0} d_{1}^{2} y^{9}+3331701 b_{0}^{2} d_{1}^{2} y^{9} \\
& +7827 a_{0}^{3} d_{1}^{3} y^{9}+50215 a_{0}^{2} b_{0} d_{1}^{3} y^{9} \\
& \left.+119361 a_{0} b_{0}^{2} d_{1}^{3} y^{9}+108056 b_{0}^{3} d_{1}^{3} y^{9}\right), \\
& u_{10}=-d_{1}\left(21952 x^{10}-3008 a_{0} d_{1} x^{10}-992 b_{0} d_{1} x^{10}\right. \\
& -605184 x^{9} y+107232 a_{0} d_{1} x^{9} y+42144 b_{0} d_{1} x^{9} y \\
& -1104 a_{0}^{2} d_{1}^{2} x^{9} y-752 a_{0} b_{0} d_{1}^{2} x^{9} y-160 b_{0}^{2} d_{1}^{2} x^{9} y \\
& +8513712 x^{8} y^{2}-1841888 a_{0} d_{1} x^{8} y^{2} \\
& -822896 b_{0} d_{1} x^{8} y^{2}+34440 a_{0}^{2} d_{1}^{2} x^{8} y^{2} \\
& +29616 a_{0} b_{0} d_{1}^{2} x^{8} y^{2}+7896 b_{0}^{2} d_{1}^{2} x^{8} y^{2} \\
& -81848832 x^{7} y^{3}+20765520 a_{0} d_{1} x^{7} y^{3} \\
& +10179424 b_{0} d_{1} x^{7} y^{3}-527376 a_{0}^{2} d_{1}^{2} x^{7} y^{3} \\
& -530448 a_{0} b_{0} d_{1}^{2} x^{7} y^{3}-162816 b_{0}^{2} d_{1}^{2} x^{7} y^{3} \\
& +1050 a_{0}^{3} d_{1}^{3} x^{7} y^{3}+1774 a_{0}^{2} b_{0} d_{1}^{3} x^{7} y^{3} \\
& +1102 a_{0} b_{0}^{2} d_{1}^{3} x^{7} y^{3}+250 b_{0}^{3} d_{1}^{3} x^{7} y^{3} \\
& +602777304 x^{6} y^{4}-173386212 a_{0} d_{1} x^{6} y^{4} \\
& -90723084 b_{0} d_{1} x^{6} y^{4}+5270270 a_{0}^{2} d_{1}^{2} x^{6} y^{4} \\
& +5948592 a_{0} b_{0} d_{1}^{2} x^{6} y^{4}+2010036 b_{0}^{2} d_{1}^{2} x^{6} y^{4}
\end{aligned}
$$




$$
\begin{aligned}
& -21020 a_{0}^{3} d_{1}^{3} x^{6} y^{4}-41960 a_{0}^{2} b_{0} d_{1}^{3} x^{6} y^{4} \\
& -31132 a_{0} b_{0}^{2} d_{1}^{3} x^{6} y^{4}-8640 b_{0}^{3} d_{1}^{3} x^{6} y^{4} \\
& -3601377936 x^{5} y^{5}+1131810888 a_{0} d_{1} x^{5} y^{5} \\
& +620482512 b_{0} d_{1} x^{5} y^{5}-38357066 a_{0}^{2} d_{1}^{2} x^{5} y^{5} \\
& -47295826 a_{0} b_{0} d_{1}^{2} x^{5} y^{5}-17155148 b_{0}^{2} d_{1}^{2} x^{5} y^{5} \\
& +212538 a_{0}^{3} d_{1}^{3} x^{5} y^{5}+481702 a_{0}^{2} b_{0} d_{1}^{3} x^{5} y^{5} \\
& +405182 a_{0} b_{0}^{2} d_{1}^{3} x^{5} y^{5}+128818 b_{0}^{3} d_{1}^{3} x^{5} y^{5} \\
& -85 a_{0}^{4} d_{1}^{4} x^{5} y^{5}-340 a_{0}^{3} b_{0} d_{1}^{4} x^{5} y^{5} \\
& -510 a_{0}^{2} b_{0}^{2} d_{1}^{4} x^{5} y^{5}-340 a_{0} b_{0}^{3} d_{1}^{4} x^{5} y^{5} \\
& -85 b_{0}^{4} d_{1}^{4} x^{5} y^{5} \\
& +17877283056 x^{4} y^{6}-5874570084 a_{0} d_{1} x^{4} y^{6} \\
& -3333151404 b_{0} d_{1} x^{4} y^{6}+212121436 a_{0}^{2} d_{1}^{2} x^{4} y^{6} \\
& +280352816 a_{0} b_{0} d_{1}^{2} x^{4} y^{6}+107174808 b_{0}^{2} d_{1}^{2} x^{4} y^{6} \\
& -1401156 a_{0}^{3} d_{1}^{3} x^{4} y^{6}-3518659 a_{0}^{2} b_{0} d_{1}^{3} x^{4} y^{6} \\
& -3250715 a_{0} b_{0}^{2} d_{1}^{3} x^{4} y^{6}-1130262 b_{0}^{3} d_{1}^{3} x^{4} y^{6} \\
& +1085 a_{0}^{4} d_{1}^{4} x^{4} y^{6}+5094 a_{0}^{3} b_{0} d_{1}^{4} x^{4} y^{6} \\
& +9160 a_{0}^{2} b_{0}^{2} d_{1}^{4} x^{4} y^{6}+7498 a_{0} b_{0}^{3} d_{1}^{4} x^{4} y^{6} \\
& +2363 b_{0}^{4} d_{1}^{4} x^{4} y^{6}-73199185920 x^{3} y^{7} \\
& +23982302232 a_{0} d_{1} x^{3} y^{7} \\
& -64886985 a_{0} b_{0}^{2} d_{1}^{3} x^{2} y^{8}-25527395 b_{0}^{3} d_{1}^{3} x^{2} y^{8} \\
& +23205 a_{0}^{4} d_{1}^{4} x^{2} y^{8}+135806 a_{0}^{3} b_{0} d_{1}^{4} x^{2} y^{8} \\
& +313360 a_{0}^{2} b_{0}^{2} d_{1}^{4} x^{2} y^{8}+341634 a_{0} b_{0}^{3} d_{1}^{4} x^{2} y^{8} \\
& +150475 b_{0}^{4} d_{1}^{4} x^{2} y^{8}-541133408592 x y^{9} \\
& +153053040600 a_{0} d_{1} x y^{9}+93565298520 b_{0} d_{1} x y^{9} \\
& -5592047067 a_{0}^{2} d_{1}^{2} x y^{9}-8557415979 a_{0} b_{0} d_{1}^{2} x y^{9} \\
& -3635890530 b_{0}^{2} d_{1}^{2} x y^{9}+42251824 a_{0}^{3} d_{1}^{3} x y^{9} \\
& +131348672 a_{0}^{2} b_{0} d_{1}^{3} x y^{9}+147010021 a_{0} b_{0}^{2} d_{1}^{3} x y^{9} \\
& +60353479 b_{0}^{3} d_{1}^{3} x y^{9}-48844 a_{0}^{4} d_{1}^{4} x y^{9} \\
& -305846 a_{0}^{3} b_{0} d_{1}^{4} x y^{9}-760242 a_{0}^{2} b_{0}^{2} d_{1}^{4} x y^{9} \\
& -901682 a_{0} b_{0}^{3} d_{1}^{4} x y^{9}-438506 b_{0}^{4} d_{1}^{4} x y^{9} \\
& +634052720592 y^{10}-159806071620 a_{0} d_{1} y^{10} \\
& -99249887196 b_{0} d_{1} y^{10}+5639205735 a_{0}^{2} d_{1}^{2} y^{10} \\
& +8832784710 a_{0} b_{0} d_{1}^{2} y^{10}+3813012729 b_{0}^{2} d_{1}^{2} y^{10} \\
& -42181381 a_{0}^{3} d_{1}^{3} y^{10}-135185562 a_{0}^{2} b_{0} d_{1}^{3} y^{10} \\
& -155218542 a_{0} b_{0}^{2} d_{1}^{3} y^{10}-64874815 b_{0}^{3} d_{1}^{3} y^{10} \\
& +48844 a_{0}^{4} d_{1}^{4} y^{10}+318016 a_{0}^{3} b_{0} d_{1}^{4} y^{10} \\
& +824332 a_{0}^{2} b_{0}^{2} d_{1}^{4} y^{10}+1024232 a_{0} b_{0}^{3} d_{1}^{4} y^{10} \\
& \left.+525776 b_{0}^{4} d_{1}^{4} y^{10}\right) \text {. }
\end{aligned}
$$$$
+13978222776 b_{0} d_{1} x^{3} y^{7}
$$$$
-892808908 a_{0}^{2} d_{1}^{2} x^{3} y^{7}-1249355132 a_{0} b_{0} d_{1}^{2} x^{3} y^{7}
$$$$
-497385264 b_{0}^{2} d_{1}^{2} x^{3} y^{7}+6463496 a_{0}^{3} d_{1}^{3} x^{3} y^{7}
$$$$
+17699203 a_{0}^{2} b_{0} d_{1}^{3} x^{3} y^{7}+17662897 a_{0} b_{0}^{2} d_{1}^{3} x^{3} y^{7}
$$$$
+6564610 b_{0}^{3} d_{1}^{3} x^{3} y^{7}-6527 a_{0}^{4} d_{1}^{4} x^{3} y^{7}
$$$$
-34734 a_{0}^{3} b_{0} d_{1}^{4} x^{3} y^{7}-72008 a_{0}^{2} b_{0}^{2} d_{1}^{4} x^{3} y^{7}
$$$$
-69410 a_{0} b_{0}^{3} d_{1}^{4} x^{3} y^{7}-26441 b_{0}^{4} d_{1}^{4} x^{3} y^{7}
$$$$
+237058519752 x^{2} y^{8}-73717828776 a_{0} d_{1} x^{2} y^{8}
$$$$
-44037236592 b_{0} d_{1} x^{2} y^{8}+2751173532 a_{0}^{2} d_{1}^{2} x^{2} y^{8}
$$$$
+4044825060 a_{0} b_{0} d_{1}^{2} x^{2} y^{8}
$$$$
+1668601218 b_{0}^{2} d_{1}^{2} x^{2} y^{8}
$$$$
-20693125 a_{0}^{3} d_{1}^{3} x^{2} y^{8}-60906347 a_{0}^{2} b_{0} d_{1}^{3} x^{2} y^{8}
$$

\section{Conflict of Interests}

The authors declare that there is no conflict of interests regarding the publication of this paper.

\section{Acknowledgments}

This work was supported by Scientific Research Foundation of Guangxi Education Department (no. ZD2014131), Research Foundation of Hezhou University (no. HZUBS201302), and Natural Science Foundation of China grants nos. 11261013 and 11371373.

\section{References}

[1] C. Sparrow, The Lorenz Equations: Bifcations, Chaos, and Strange Attractors, Springer, New York, NY, USA, 1982.

[2] T. Li, G. Chen, and Y. Tang, "On stability and bifurcation of Chen's system," Chaos, Solitons and Fractals, vol. 19, no. 5, pp. 1269-1282, 2004. 
[3] L. F. Mello and S. F. Coelho, "Degenerate Hopf bifurcations in the Lü system," Physics Letters A, vol. 373, no. 12-13, pp. 1116$1120,2009$.

[4] M. Messias, D. D. C. Braga, and L. F. Mello, "Degenerate Hopf bifurcations in Chua's system," International Journal of Bifurcation and Chaos in Applied Sciences and Engineering, vol. 19, no. 2, pp. 497-515, 2009.

[5] J. Hofbauer and J. W.-H. So, "Multiple limit cycles for threedimensional Lotka-Volterra equations," Applied Mathematics Letters, vol. 7, no. 6, pp. 65-70, 1994.

[6] Z. Lu and Y. Luo, "Two limit cycles in three-dimensional LotkaVolterra systems," Computers \& Mathematics with Applications, vol. 44, no. 1-2, pp. 51-66, 2002.

[7] M. Gyllenberg and M. Yan, "Four limit cycles for a threedimensional competitive Lotka-Volterra system with a heteroclinic cycle," Computers \& Mathematics with Applications, vol. 58, no. 4, pp. 649-669, 2009.

[8] Q. Wang, W. Huang, and B. Li, "Limit cycles and singular point quantities for a 3D Lotka-Volterra system," Applied Mathematics and Computation, vol. 217, no. 21, pp. 8856-8859, 2011.

[9] Q. Wang, Y. Liu, and H. Chen, "Hopf bifurcation for a class of three-dimensional nonlinear dynamic systems," Bulletin des Sciences Mathématiques, vol. 134, no. 7, pp. 786-798, 2010.

[10] J. Carr, Applications of Centre Manifold Theory, vol. 35 of Applied Mathematical Sciences, Springer, New York, NY, USA, 1981.

[11] M. J. Alvarez and A. Gasull, "Generating limit cycles from a nilpotent critical point via normal forms," Journal of Mathematical Analysis and Applications, vol. 318, no. 1, pp. 271-287, 2006.

[12] M. J. Álvarez and A. Gasull, "Monodromy and stability for nilpotent critical points," International Journal of Bifurcation and Chaos, vol. 15, no. 4, pp. 1253-1265, 2005.

[13] J. Chavarriga, H. Giacomin, J. Gine, and J. Llibre, "Local analytic integrability for nilpotent centers," Ergodic Theory and Dynamical Systems, vol. 23, no. 2, pp. 417-428, 2003.

[14] R. Moussu, "Symetrie et forme normale des centres et foyers degeneres," Ergodic Theory and Dynamical Systems, vol. 2, no. 2, pp. 241-251, 1982.

[15] A. F. Andreev, A. P. Sadovskii, and V. A. Tsikalyuk, "The centerfocus problem for a system with homogeneous nonlinearities in the case of zero eigenvalues of the linear pact," Differential Equations, vol. 39, no. 2, pp. 155-164, 2003.

[16] H. Giacomini, J. Giné, and J. Llibre, "The problem of distinguishing between a center and a focus for nilpotent and degenerate analytic systems," Journal of Differential Equations, vol. 227, no. 2, pp. 406-426, 2006.

[17] Y. Liu and J. Li, "Bifurcations of limit cycles and center problem for a class of cubic nilpotent system," International Journal of Bifurcation and Chaos in Applied Sciences and Engineering, vol. 20, no. 8, pp. 2579-2584, 2010.

[18] Y. Liu and J. Li, "On third-order nilpotent critical points: integral factor method," International Journal of Bifurcation and Chaos, vol. 21, no. 5, pp. 1293-1309, 2011.

[19] F. Li, Y. Liu, and Y. Wu, "Center conditions and bifurcation of limit cycles at three-order nilpotent critical point in a seventh degree Lyapunov system," Communications in Nonlinear Science and Numerical Simulation, vol. 16, no. 6, pp. 2598-2608, 2011.

[20] A. Buică, I. A. García, and S. Maza, "Existence of inverse Jacobi multipliers around Hopf points in $\mathbb{R}^{3}$ : emphasis on the center problem," Journal of Differential Equations, vol. 252, no. 12, pp. 6324-6336, 2012.
[21] V. F. Edneral, A. Mahdi, V. G. Romanovski, and D. S. Shafer, "The center problem on a center manifold in $\mathbb{R}^{3}$," Nonlinear Analysis, vol. 75, no. 4, pp. 2614-2622, 2012.

[22] F. S. Dias and L. F. Mello, "Analysis of a quadratic system obtained from a scalar third order differential equation," Electronic Journal of Differential Equations, vol. 2010, 6 pages, 2010.

[23] J. Llibre, "On the integrability of the differential systems in dimension two and of the polynomial differential systems in arbitrary dimension," The Journal of Applied Analysis and Computation, vol. 1, no. 1, pp. 33-52, 2011. 


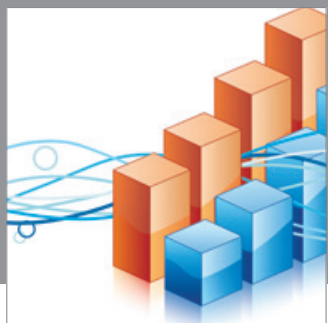

Advances in

Operations Research

mansans

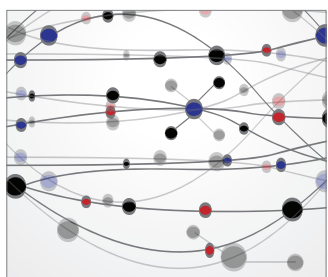

The Scientific World Journal
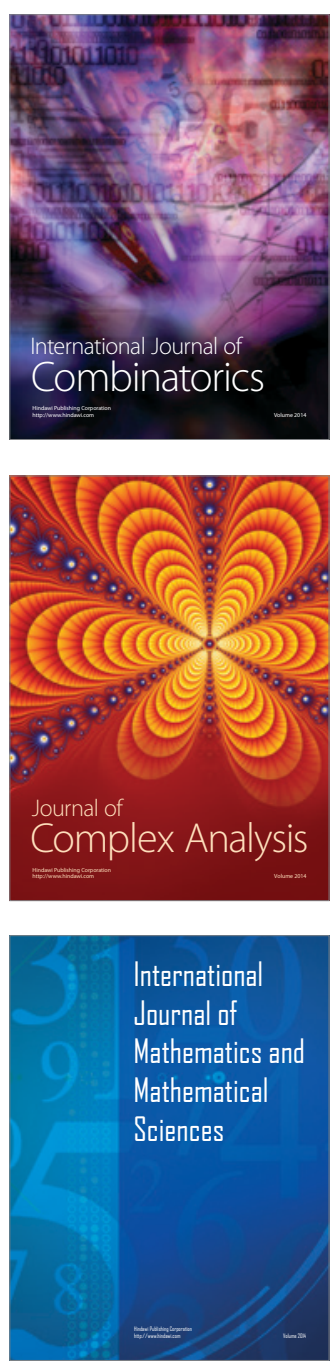
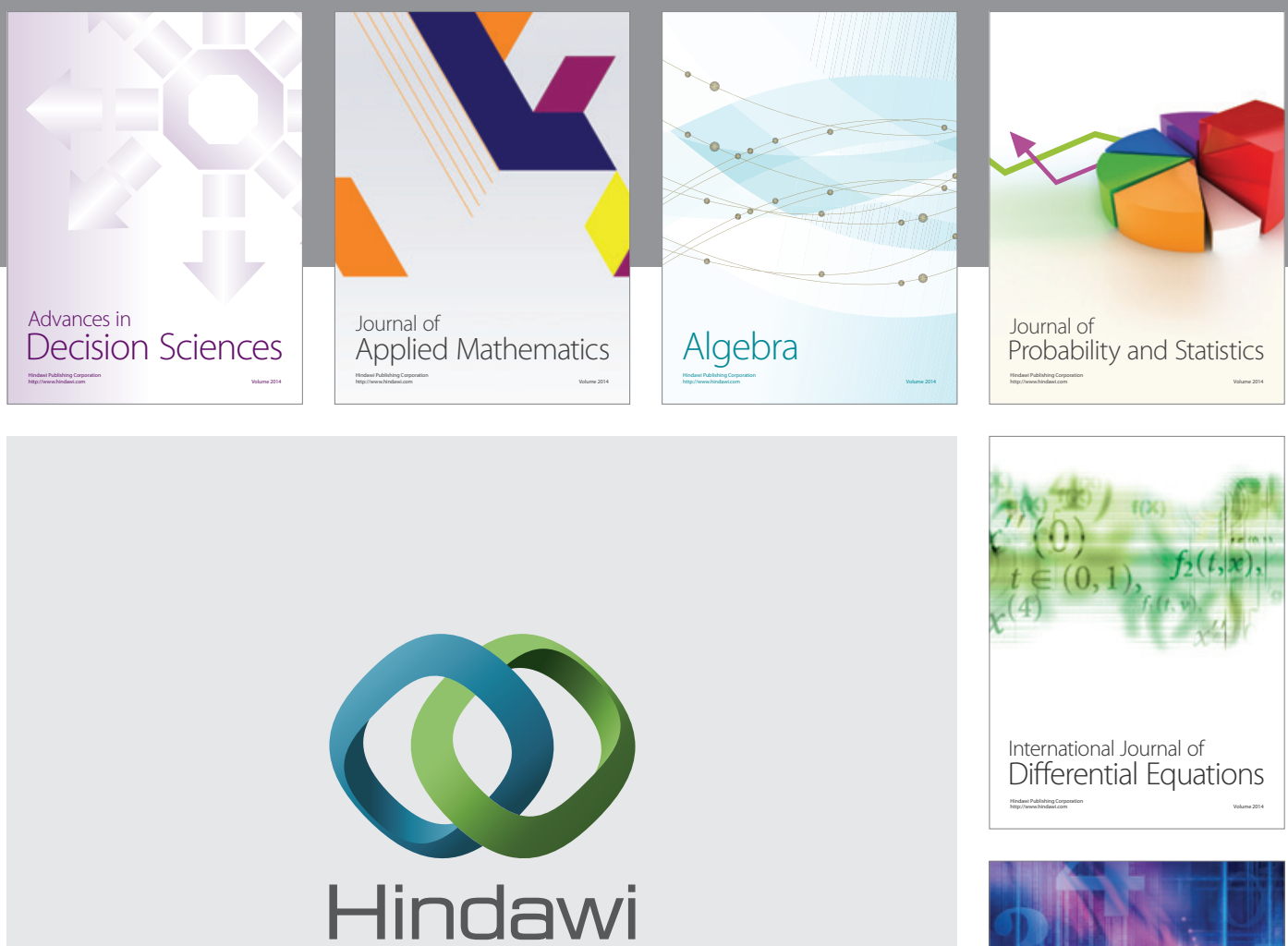

Submit your manuscripts at http://www.hindawi.com
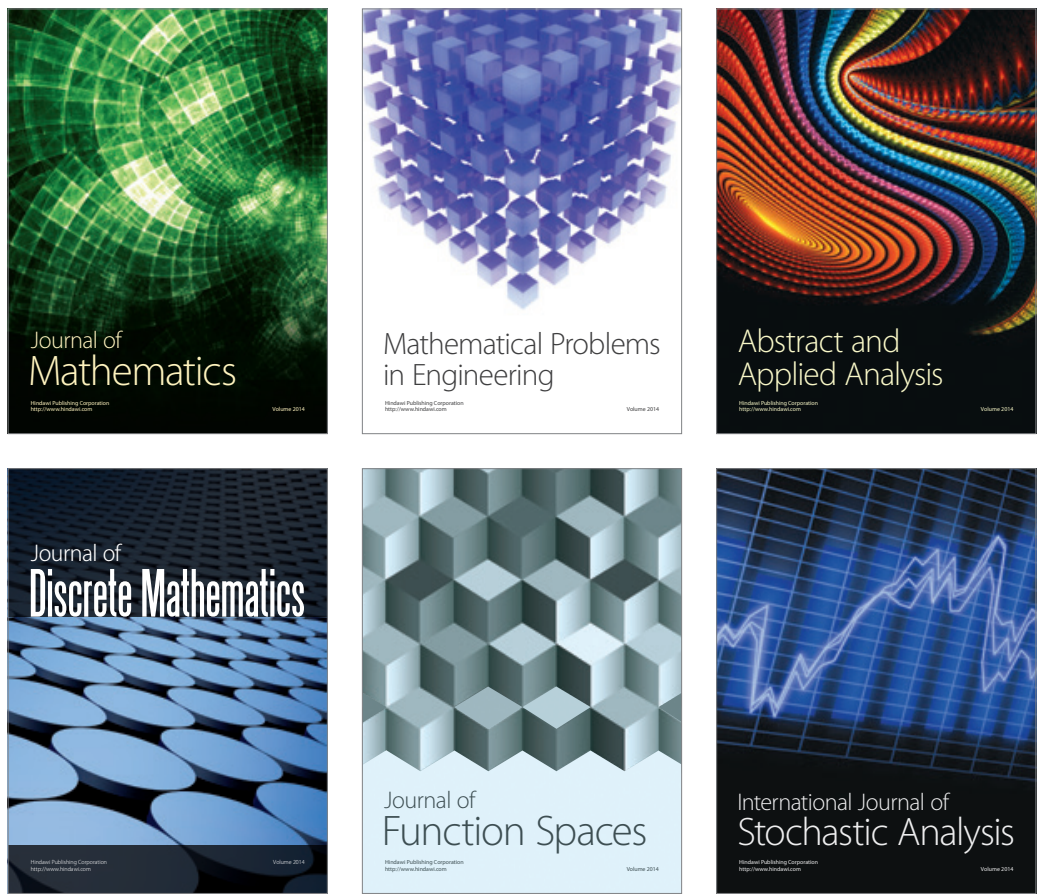

Journal of

Function Spaces

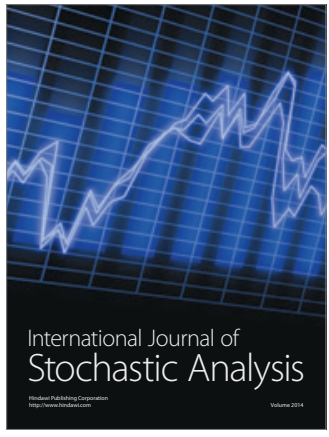

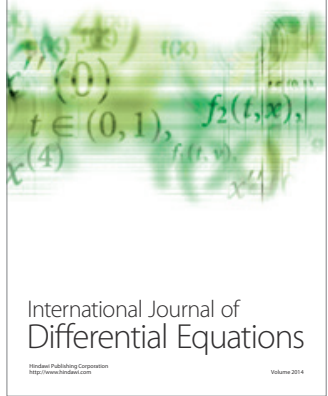
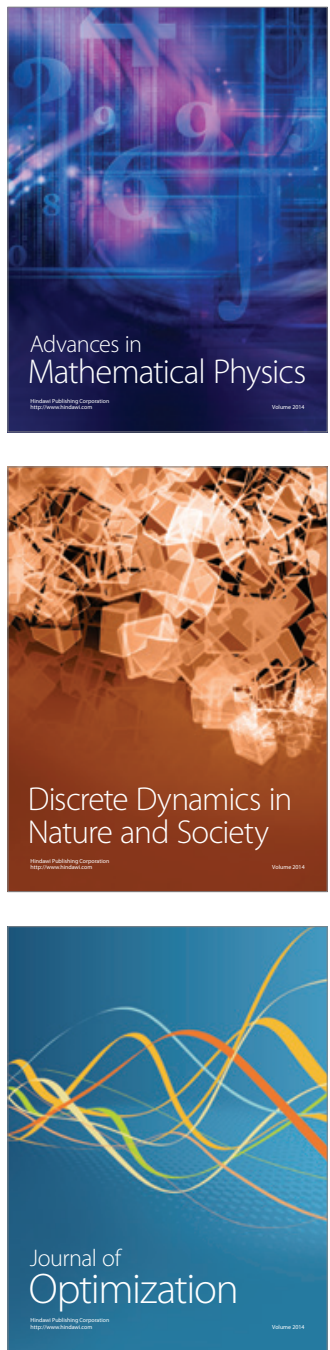